\title{
Guerre et assemblée, découverte et apprentissage de la démocratie chez les Miskitus nicaraguayens (1981-1988)
}

War and Assembly, the discovery and the learning of democracy by the Nicaraguan Miskitus (1981-1988)

\section{Gilles Bataillon}

\section{QpenEdition Journals}

\section{Édition électronique}

URL : http://journals.openedition.org/conflits/846

DOI : $10.4000 /$ conflits.846

ISSN : $1777-5345$

\section{Éditeur :}

CCLS - Centre d'études sur les conflits lilberté et sécurité, L'Harmattan

\section{Édition imprimée}

Date de publication : 1 septembre 2002

Pagination : 195-215

ISBN : 2-7475-3432-4

ISSN : 1157-996X

\section{Référence électronique}

Gilles Bataillon, « Guerre et assemblée, découverte et apprentissage de la démocratie chez les Miskitus nicaraguayens (1981-1988) », Cultures \& Conflits [En ligne], 47 | automne 2002, mis en ligne le 29 avril 2003, consulté le 30 mars 2021. URL : http://journals.openedition.org/conflits/846 ; DOI : https://doi.org/10.4000/conflits.846

Ce document a été généré automatiquement le 30 mars 2021.

Creative Commons License 


\title{
Guerre et assemblée, découverte et apprentissage de la démocratie chez les Miskitus nicaraguayens (1981-1988)
}

\author{
War and Assembly, the discovery and the learning of democracy by the \\ Nicaraguan Miskitus (1981-1988)
}

Gilles Bataillon

Jusqu'en 1979, les Miskitus nicaraguayens ont connu un entrelacement de formes politiques que l'on pourrait qualifier de traditionnelles selon la terminologie weberienne ${ }^{1}$. Vivant sur un territoire de près de $40000 \mathrm{~km} 2$, les actuelles régions autonomes de l'Atlantique, ces 120000 indiens ont connu une histoire politique peu commune à l'échelle amérindienne. Alliés des pirates français et anglais aux XVIe et XVIIe siècles, ils ont par la suite constitué un Royaume indépendant de la Moskitia, dont le souverain reconnaissait l'autorité de la Couronne britannique. En 1894, l'intégration des Miskitus au Nicaragua à la suite de longues tractations entre Britanniques, Nord-américains et Nicaraguayens, ne les a pas pour autant conduits à abandonner leurs particularismes. Convertis à la religion morave au milieu du XIXe siècle, ils résidaient, pour leur immense majorité, dans des villages dont la taille variait entre 200 à 2000 habitants. Si leurs villages faisaient partie de circonscriptions administratives nicaraguayennes, s'ils étaient formellement soumis à des autorités désignées par le pouvoir central (jueces de mesta), ou élues au cours d'élections pour le moins frauduleuses, les maires et les députés se pensaient de fait avant tout comme les membres de communautés politiques indépendantes. Si beaucoup d'entre eux partaient travailler comme saisonniers dans les plantations de café, ou comme mineurs dans les montagnes du centre du pays, ou encore comme marins sur des navires de pêche, cette intégration de longue date aux circuits de l'économie mondiale ne les empêchait nullement de préserver l'usage de leur langue et de coutumes propres. Ces communautés vivaient de fait avant tout sous l'autorité conjointe des pasteurs, des 
anciens de la congrégation, des anciens du village. Leurs idéaux consistaient à respecter les normes de l'Eglise morave sous l'autorité d'un clergé autochtone, devenu officiellement indépendant en 1949 des missionnaires venus d'Allemagne puis des Etats-Unis. C'est dire que deux traditions, pour une part contradictoires, étaient également valorisées. En effet, si la lettre de la Bible constituait à n'en pas douter l'ultima ratio, toute une tradition protestante des assemblées et d'une libre discussion en leur sein apparaissait simultanément comme l'idéal même de la vie chrétienne. Ce goût de la liberté de parole s'accompagnait d'une valorisation des décisions prises en commun au terme d'interminables palabres. Il n'était toutefois nullement question de décisions prises à la majorité des voix après délibération, et encore moins de majorité et d'opposition. Les discussions collectives devaient conduire à des actions décidées à l'unanimité. Et une fois celle-ci atteinte, on ne devait plus parler publiquement que d'une seule voix, ce tant face à d'autres communautés, que face aux agents du pouvoir central.

Depuis 1989, date de la fin de la guerre civile contras/sandinistes et de la victoire de l'opposition sur le Front sandiniste de libération nationale (FSLN) ${ }^{2}$, les Miskitus font l'expérience à tous les niveaux de la vie sociale de formes de pouvoir démocratiques. Ce sont désormais toutes les autorités municipales - maires et conseillers municipaux, comme les députés et les membres des conseils régionaux autonomes - qui sont élues au suffrage universel. Ce système de désignation des autorités ou des représentants à l'issue d'une compétition électorale s'est généralisé à tous les niveaux. Ainsi dans les communautés, si petites soient-elles, les syndics et les juges communautaires sont, eux aussi, élus de la façon la plus formelle. Mieux, les anciens qui s'imposaient autrefois par leur âge et leur expérience sont aujourd'hui remplacés par des autorités locales élues qui portent le même nom et qui font aussi partie d'un Conseil régional des anciens. On constate parallèlement une érosion considérable du pouvoir d'influence et de contrôle social de l'Eglise morave. Celle-ci ne doit pas seulement affronter la concurrence des Eglises pentecôtistes. Se fait jour l'idée que les Eglises n'ont plus le dernier mot sur l'ordre du monde et que leurs commandements sont susceptibles d'être discutés, et que chacun est désormais son propre juge ${ }^{3}$. D'une façon plus générale, le credo chrétien est battu en brèche par la découverte de la thématique des droits de l'homme et de l'égalité des conditions.

La découverte et l'apprentissage de pratiques et de mœurs démocratiques par les Miskitus tiennent sans nul doute à «l'esprit du temps». Ils s'inscrivent de toute évidence dans un mouvement plus large de la valorisation de la démocratie qui a marqué l'ensemble du Nicaragua et plus généralement le sous-continent depuis le début des années 1980. Pourtant, cette découverte et cet apprentissage ont aussi été des expériences propres aux miskitus qu'il convient de retracer d'autant qu'ils prennent place dans un contexte qui leur était a priori peu favorable, celui de l'action armée et de la guerre civile. C'est en effet au cours de cette guerre que des membres de la guérilla indianiste MISURA instituèrent peu à peu des pratiques démocratiques tant au sein de leur organisation armée que des populations déplacées et réfugiées au Honduras. Retracer les différents moments de cette invention et la mise en place de praxis démocratiques, tel est l'objet de cette étude. Je commencerai par décrire le contexte qui prévaut à l'issue des deux premières années d'affrontements armés ainsi que les formes du pouvoir au sein de la guérilla. Ceci pour montrer ensuite comment c'est à la croisée des nécessités fonctionnelles de la guerre, de l'érosion du charisme du principal dirigeant de la guérilla et de certaines pressions de l'opinion publique internationale 
que virent le jour les premières pratiques démocratiques. J'analyserai également les différents moments de la mise en place de cette sociabilité démocratique et les conséquences de cette réorganisation tant sur la manière de conduire la guerre que dans les rapports avec les réfugiés.

De l'épuisement du charisme aux " prises de parole ${ }^{4}$ »

Si les premiers moments des opérations armées contre les sandinistes se sont déroulés dans une ambiance marquée par le pouvoir discrétionnaire du commandant en chef de MISURA, les aléas de la guerre ne vont pas tarder à favoriser des "prises de parole " tous azimuts. On assiste ainsi tout à la fois à l'épuisement et à la remise en cause du pouvoir charismatique de Steadman Fagoth, au surgissement d'un contre-pouvoir des comandantes. Parallèlement, fortes de l'appui du Haut Commissariat aux Réfugiés (H.C.R.), les populations déplacées acquièrent une autonomie et se dotent d'institutions représentatives propres face aux guérilleros.

Ayant vu ailleurs ${ }^{5}$ comment le piétinement des opérations armées allié à la suspension de l'aide nord-américaine ont favorisé l'émergence d'un contre-pouvoir au sein de la guérilla, il convient maintenant d'en décrire et d'en analyser le fonctionnement. Alors que dans les premiers temps de la guerre, les guérilleros obéissaient sans discuter aux ordres de l'Etat-major, les comandantesont de facto obtenu d'être associés à la planification des opérations militaires. Les opérations sont dorénavant analysées et discutées par les comandantes et l'Etat-major avant d'être lancées. C'est dire que si certaines sont adoptées sans grandes modifications, d'autres sont longuement discutées et redéfinies avec les intéressés. De même, les comandantes ont-ils acquis de réelles marges d'initiative et n'hésitent plus à faire part de leurs souhaits comme de leurs veto. Certains entrent même en contact avec les membres de la guérilla indienne rivale, MISURASATA, dont l'Etat-major est basé au Costa Rica, et commencent à monter des opérations en commun avec eux. Si ce contre-pouvoir des comandantes n'est au début qu'un contre-pouvoir de fait et nullement de droit, il n'en transforme pas moins complètement les modalités de fonctionnement du pouvoir au sein de l'organisation armée. L'Etat-major n'apparaît plus comme un pouvoir omniscient dont on ne saurait discuter les ordres, mais au contraire comme une instance avec laquelle on est d'une certaine façon en négociation permanente. A la différence des deux premières années de guerre (1982-1983), de telles discussions ne font plus figures de "trahisons» passibles de la peine capitale. Elles acquièrent au contraire un aspect quasi routinier. Mieux, nombre de combattants ne tardent pas à découvrir qu'elles ont une valeur fonctionnelle. En effet, qui mieux qu'un comandante familier d'une zone peut juger de la faisabilité d'une attaque ou d'un coup de main. Si de telles conduites ne sont au départ que le fait d'une poignée de comandantes, ces manières ne tardent pas à faire tache d'huile. Ce sont en effet un nombre chaque fois plus grand de responsables qui s'autorisent des marges d'autonomie croissante. Ce sont aussi des sous-officiers et de simples guérilleros qui adoptent la même attitude vis-à-vis des comandantes. Si l'on ne saurait exagérer la portée de ce changement, il ne s'agit d'aucune façon de la mise en place d'un système de démocratie directe au sein d'une organisation militaire, il n'en convient pas moins de souligner qu'un autre style de relations d'obéissance et de commandement se fait jour au sein de MISURA. Fagoth et son Etat-major sont désormais dans l'obligation de présenter leurs options aux comandanteset de les discuter avec eux comme peuvent le faire un révérend ou un pasteur avec les membres du Conseil des anciens de leur paroisse. 
En même temps, l'arrivée de milliers de réfugiés au début 1981 et leur prise en charge par le H.C.R. va largement peser en faveur d'un style de relations plus démocratique. La charte des Nations Unies donne en effet au H.C.R. de réels pouvoirs de protection des populations qu'il prend en charge. Les camps placés sous son autorité sont autant d'enclaves soustraites à l'emprise des pouvoirs locaux qu'il s'agisse de ceux de l'armée hondurienne ou de ceux de la guérilla. De plus, les membres des organisations humanitaires qui travaillent auprès des réfugiés pour le compte du H.C.R. sont le plus souvent très favorables aux sandinistes. Car quelles que soient les réserves de certains devant les «ratés » de la révolution, pratiquement personne ne nourrit de sympathie pour les guérilleros de MISURA. Aussi sont-ils très réceptifs aux plaintes de tous ceux qui protestent contre le véritable système de conscription, très souvent forcée, qu'ont mis en place les guérilleros. Du coup, quelles que soient les pressions des militaires honduriens, les camps du H.C.R. vont pouvoir servir de lieux de refuge, tant à ceux qui ont fui les exactions des militaires sandinistes, qu'à ceux qui refusent le nouveau pouvoir des guérilleros. Et bien que les cas de coactions de la guérilla au sein des camps restent nombreux, les autorités du H.C.R. n'en sont pas moins susceptibles de fournir un réel appui à certains opposants à la guérilla. Sans jamais vraiment cesser, les abus des guérilleros se font du coup plus discrets, et ceux qui entendent s'y soustraire acquièrent une nouvelle marge de manœuvre ${ }^{6}$.

Désireux d'affirmer son emprise sur les réfugiés, le H.C.R. va aussi les pousser à élire démocratiquement des autorités représentatives. Chaque communauté est ainsi invitée à désigner une junta directiva coordinadora composée de sept membres : un coordinateur, un juge, un secrétaire, un trésorier, un fiscal, et un ou plusieurs porte-parole selon la taille de la communauté. Dans ce dessein, les membres des O.N.G. travaillant pour le H.C.R. convoquent à des assemblées générales dans chaque communauté. Les comunitarios qui désirent se porter candidats aux différents postes se font connaître. Après quoi, l'ensemble des adultes de la communauté se met en file pour voter. Chacun d'entre eux fait part publiquement de ses choix aux membres du H.C.R. qui les notent sur un tableau noir. Enfin, à l'issue du décompte des suffrages ainsi exprimés, on proclame le résultat de l'élection des autorités. Une fois ces juntas élues, le H.C.R. désigne certains de leurs membres pour faire partie d'un Comité de travail chargé de faire le lien entre ses représentants et les membres de ces juntas. S'il ne fait nul doute que des membres de MISURA - comme des proches de la guérilla - occuperont nombre de ces postes, ils n'en auront pas moins été désignés au cours d'élections organisées par une institution qui leur est pour le moins défavorable. Et quelles que soient les possibilités d'intimider de possibles candidats qui leur seraient par trop hostiles, les guérilleros ne peuvent nullement empêcher l'apparition et l'expression publique de dissensions et de rivalités au sein de la nébuleuse des exilés. De même, bien que les préférences ne s'expriment pas secrètement, personne n'est assuré du résultat du scrutin. Ainsi, quelle que soit l'influence de MISURA au sein des camps de réfugiés, les exilés acquièrent peu à peu une réelle autonomie face au pouvoir de fait de Steadman Fagoth et de son Etat-major.

Enfin, en 1984, la baisse d'intensité des opérations militaires, consécutive à la suspension de l'aide nord-américaine, va donner l'occasion à l'ensemble des combattants de s'interroger sur le sens de leurs actions. On assiste ainsi tout à la fois à des discussions informelles au sein de la troupe comme à des consultations plus organisées. Beaucoup des guérilleros se sont en effet jetés dans l'action pensant qu'ils seraient rapidement vainqueurs. Si les succès se sont fait attendre, l'aide nord- 
américaine leur a toutefois permis de survivre en 1982 et en 1983 dans des conditions acceptables. Celle-ci suspendue, les discussions sur le sens de leur combat et leur futur immédiat deviennent d'une certaine façon inévitables.

Ces débats vont connaître un regain d'intensité en juin 1984 lors d'un séminaire de formation des cadres de MISURASA. Alors que les participants à ce séminaire étaient d'ordinaire séparés géographiquement, ils vont pouvoir pour la première fois se retrouver et discuter à foison des modalités du fonctionnement du mouvement comme $\mathrm{du}$ futur de la guerre. Les échanges avec des anthropologues et des journalistes venus enquêter sur MISURA - parfois très critiques à l'encontre de certaines de leurs actions contribueront aussi à renforcer le sentiment de beaucoup d'entre eux d'avoir leur mot à dire sur la conduite des opérations politico-militaires. Les discussions avec ceux-ci vont en effet d'abord porter tout à la fois sur leurs chances de l'emporter militairement sur les sandinistes, sur la nécessité de négocier avec ces derniers et sur la nature de leurs relations avec la Contra hispanophone. Elles porteront ensuite sur leurs rapports avec les populations civiles, comme sur la question des recrutements forcés et celle des exactions et de certains assassinats dont furent victimes des guérilleros discutant l'autorité de Fagoth et de son Etat-major.

$\mathrm{Au}$ lendemain de ce séminaire, les comandantes vont très ouvertement reprendre ces discussions avec leurs troupes. Ils débattront ainsi des modalités de leur survie comme du futur de la lutte armée. Pour ce qui est de leur survie, les combattants ont le choix entre trois options. Ils peuvent se rendre dans les camps de réfugiés et accepter une démobilisation de fait en attendant mieux. D'autres décident à l'inverse de s'établir sur la rive hondurienne du fleuve Frontalier, le Rio Coco. Ce faisant, ils vivent avec leurs familles dans une incontestable précarité mais à distance des pressions des militaires honduriens ou de certains discours moralisateurs des membres des O.N.G. travaillant pour le H.C.R. La troisième option consiste pour quelques combattants particulièrement aguerris à nomadiser à l'intérieur du territoire nicaraguayen, en se ravitaillant dans différents villages où ils comptent des partisans. Par ailleurs, différents comandantes au contact avec l'organisation rivale, MISURASATA, décident d'engager des pourparlers avec leurs anciens compagnons d'armes ${ }^{7}$.

Parallèlement, certains comandantes entreprennent de renouer les liens avec les réfugiés et leurs nouvelles autorités élues. Attentifs aux doléances et aux critiques des réfugiés, ils reconnaissent comme autant d'autorités légitimes les membres des juntas avec lesquels ils décident de présenter différentes propositions de travail au H.C.R. Et, en quelques mois, le Comité de travail jugé trop docile face aux membres du H.C.R. est remplacé par un Comité central élu au sein des membres des juntas.

L'année 1984 est donc marquée par toute une série d'incertitudes qui conduisent à la fois à une remise en cause du pouvoir charismatique de Steadman Fagoth, mais aussi à un processus de réflexion tous azimuts sur les sens de la lutte contre la tyrannie sandiniste, comme sur le mode d'organisation des réfugiés. Dorénavant rares sont ceux qui, au sein de la guérilla comme au sein des réfugiés, pensent qu'il est des dirigeants capables de mener seuls la guerre avec les sandinistes. Se fait peu à peu jour l'idée d'une nécessaire direction collégiale des opérations, comme de l'importance d'agir avec l'assentiment du plus grand nombre et de réunifier les deux guérillas indiennes antisandinistes.

L'invention de nouvelles institutions et leur mise en place 
La mise en place de nouvelles institutions politico-militaires va être grandement favorisée par les circonstances. En effet, quels que soient leur activisme et leur capacité à convaincre un nombre croissant de guérilleros et de réfugiés de la nécessité d'une " réforme ", les opposants à Fagoth se heurtent à un obstacle de taille. Le commandant en chef de MISURA bénéficie de l'appui des autorités militaires honduriennes qui sont toutes puissantes dans la zone frontalière. Car, bien que les temps aient changé, qu'il doive faire place à certaines demandes des comandantes et qu'il ne puisse plus aussi facilement faire assassiner les opposants comme dans les premiers temps de la guerre, il garde néanmoins le pouvoir de les faire expulser par les forces armées honduriennes. Les militaires honduriens sont d'autant plus complaisants avec lui qu'il a accepté qu'ils détournent à leur profit une bonne partie de l'aide versée par les Etats-Unis. Quels que soient les aménagements possibles au sein de MISURASA, il ne saurait être question de remettre en cause le statut politico-militaire de Fagoth et encore moins de l'écarter par un coup de force. C'est paradoxalement les militaires honduriens qui vont permettre sa mise sur la touche. Ceux-ci sont en effet en butte aux critiques de nombreuses organisations de défense des droits de l'homme qui, preuves à l'appui, les accusent de couvrir les crimes de la Contra et de pratiquer eux-mêmes nombre d'exactions contre des syndicalistes et des militants de gauche. Aussi quand en décembre 1984, au lendemain de la capture de plusieurs miliciens sandinistes, Fagoth déclare publiquement qu'il convient de les passer par les armes et de ne pas s'encombrer de prisonniers, il déclenche un tollé dans la presse hondurienne et nord-américaine. Soucieuses de se dédouaner à peu de frais, les autorités militaires honduriennes le déclarent persona non grataet l'expulsent vers les Etats-Unis.

Si le départ de Fagoth est tout à fait inattendu et crée une situation de vide du pouvoir, il n'en existe pas moins une possible relève. De fait, celle-ci est d'autant plus à même d'être reconnue que les Nord-américains et les Honduriens, qui ne souhaitent nullement la disparition de la guérilla miskitu, ont besoin d'interlocuteurs facilement identifiables. Enfin, les comandantesréformateurs peuvent d'autant mieux se faire entendre que le contexte semble leur donner raison. Leurs avertissements quant aux aspects particulièrement contre-productifs des exactions de la guérilla viennent de trouver une confirmation éclatante. Fagoth, naguère craint et en apparence tout puissant, a été purement et simplement expulsé du pays. Les partisans de ce dernier sont à la fois complètement démoralisés, certains abandonnent d'ailleurs la lutte armée, et apparaissent comme particulièrement peu crédibles. Du coup, les comandantes réformateurs vont se trouver dans une conjoncture d'opportunité tout à fait favorable pour mettre en place de nouvelles autorités.

Leur premier soin va être de convoquer l'ensemble des cadres politiques et militaires afin de désigner un nouvel Etat-major à la tête duquel siègent deux des leurs : Raul Tobias et Samuel Kitler. Ces nouvelles autorités sont considérées comme provisoires et ont à charge de convoquer une assemblée où siégeront des représentants des guérilleros et des différentes communautés. Les organisateurs de cette assemblée entendent faire œuvre de refondateurs à l'image de ceux qui furent à l'origine des premières organisations indianistes miskitus, ACARIC (1968), ALPROMISU (1974) et MISURASATA (1979). Sont convoqués à la fois tous les comandantesde MISURA, mais aussi ceux de l'organisation rivale MISURASATA. On invite aussi de la même façon des représentants des communautés exilées au Honduras et des représentants des communautés dont le gros des habitants n'a pas été déplacé par la guerre, comme les gens de la région littorale (Sandy Bay), des alentours de Puerto Cabezas ou de la région 
des mines (Siuna, Rosita et Bonanza). On invite enfin à siéger à cette assemblée des représentants des communautés créoles et sumus. Et c'est près de cinquante-six communautés, pour la plupart en exil, qui répondront à cette invitation. Ainsi, pour la première fois depuis le début de l'entrée en guerre contre les sandinistes (décembre 1981), on va voir siéger côte à côte des exilés et des gens de l'intérieur, des Miskitus, des Sumus, des créoles et des hispanophones; et des guérilleros d'organisations rivales. A la différence des précédentes assemblées, celle-ci n'est pas convoquée par des notables appuyés par des institutions surplombant la société miskitu. En effet, que ce soit lors de la fondation d'ACARIC, ou lors de celle d'ALPROMISU, pasteurs, professeurs et prêtres avaient joué un rôle moteur. Et ces deux organisations étaient pour ainsi dire nées à l'ombre de l'Eglise morave et de l'Eglise catholique. De même, MISURASATA était-elle née au forceps sous la houlette du Front sandiniste qui avait appuyé un groupe d'étudiants miskitu soucieux de jouer un rôle de premier plan aux dépens des dirigeants d'ALPROMISU. Ici rien de tel. Ce sont de jeunes comandantes, pour la plupart anciens activistes lors des campagnes d'alphabétisation en langue vernaculaire, combattants de la première heure contre les sandinistes au lendemain de l'arrestation des dirigeants de MISURASATA (18 février1981), qui sont les puissances invitantes. Le contexte et les enjeux de cette assemblée sont tout à fait exceptionnels. Il s'agit tout d'abord de rassembler, par-delà la diversité de leurs situations et du degré de leurs engagements dans l'opposition aux sandinistes, des représentants du plus grand nombre de composantes de la société miskitu. Il convient aussi de les amener à délibérer pour décider de la poursuite ou non de la guerre. Il faut enfin les amener à élire une nouvelle direction politico-militaire susceptible d'être reconnue par les différents groupes de guérilleros. Durant les premières semaines de l'année 1985 les comandantesréformateurs vont s'employer à mettre sur pied cette assemblée plénière. Les contacts sont relancés avec les communautés nicaraguayennes comme avec les guérilleros de MISURASATA. Des pourparlers sont engagés avec les militaires honduriens pour que ceux-ci ne mettent pas d'entraves à cette réunion et qu'ils facilitent le transport de certains représentants des communautés en exil, installés parfois très loin de la frontière. Des dizaines de guérilleros sont mobilisés pour construire une gigantesque paillote destinée à accueillir et à abriter les débats. De même prépare-t-on tout un stock de nourriture pour les futurs visiteurs. Ainsi, la première semaine de septembre 1985, une assemblée réunit trois jours durant à RusRus plus de 500 délégués $^{8}$ ayant le pouvoir de voter. Sont présents quelques cent comandantes, un nombre équivalent de "politiques" et trois cent soixante-cinq représentants des communautés. Si les comandantes et les "politiques» sont des gens dont le savoir faire et l'influence ont été reconnus au cours de la guerre contre les sandinistes, aussi bien au sein des guérillas que des réfugiés, aucun d'entre eux n'a pourtant été formellement désigné par des gens dont ils seraient les représentants. Ils sont autant de personnalités qui se sont imposées et qui apparaissent comme porteuses d'expériences qu'ils convient de prendre en compte dans les délibérations. Les délégués des communautés ont en revanche été désignés de façon beaucoup plus formelle. Les communautés en exil installées dans les camps du H.C.R. délégueront les trois premiers membres de leurs juntas, tandis que les autres sont invitées à envoyer trois des leurs à cette réunion. Réunis à la base de Rus-Rus, les membres de cette assemblée vont commencer par discuter deux jours durant de la situation militaire et politique. Ces discussions seront tout à la fois le moment d'échanges d'informations comme l'occasion de débats entre des personnalités représentant des options concurrentes et 
divergentes. Pour la première fois depuis 1982, non seulement les têtes de file dela guérilla, mais aussi des représentants des communautés s'interrogent ouvertement et collectivement sur le bien-fondé de la poursuite de la guerre. Et il est à noter que, selon les dires de différents participants à cette réunion, personne n'excluait la possibilité qu'une majorité se dessine en faveur de pourparlers et d'une trêve avec les sandinistes. Ces premiers échanges conduisent pourtant rapidement l'ensemble des présents à opter pour la poursuite de la guerre. Vient ensuite le moment de la désignation d'une nouvelle direction politico-militaire. Ici encore l'événement frappe par sa nouveauté. Jusqu'à présent les dirigeants d'ACARIC, comme d'ALPROMISU ou de MISURASATA, s'étaient imposés sans devoir affronter de concurrents. S'ils avaient été élus, ils l'avaient été au cours d'élections non concurrentielles. A Rus-Rus, il va au contraire y avoir une véritable compétition entre des équipes rivales pour désigner tout à la fois le directoire politique, le conseil des anciens, le conseil politique, son équipe politique et l'Etat-major ${ }^{9}$. Ainsi, il n'y aura en effet pas moins de trois listes concurrentes pour les postes de commandant en chef et de commandant en chef en second : l'une composée avant tout de comandantes issus de MISURASATA et deux autres composées de comandantesde MISURA. C'est finalement une liste MISURA, dont les promoteurs auront eu la sagesse de présenter côte à côte des guérilleros issus du Rio Coco aux côtés d'autres natifs des llanos, qui l'emportera. Comme lors de la délibération sur la poursuite de la guerre, les débats seront là aussi marqués par la plus grande incertitude. Ce n'est qu'au cours du vote où chaque participant sera appelé à exprimer publiquement et individuellement son choix que se dessinera l'issue du scrutin. Enfin, soucieux de marquer leur volonté de renouveau, les membres de l'assemblée de RusRus dissolvent les deux organisations de guérilla rivales, MISURA et MISURASATA qui apparaissent par trop inféodées à Fagoth et Rivera ; et fondent un nouveau mouvement, KISAN (Kus Indianka Sut Aslika Nicaragua-l'unité des peuples de la côte atlantique du Nicaragua), dont le nom marque la volonté de réunifier les différentes composantes de la côte atlantique nicaraguayenne.

Nul doute que l'assemblée de Rus-Rus ne révèle de sérieuses entraves au jeu démocratique. Ainsi, les délégués qui siègent et délibèrent sont pratiquement et uniquement des hommes. Car si dans les communautés les femmes ont pris part à la désignation des délégués, ce ne sont jamais elles qui ont été désignées représentantes de leurs communautés. Un tel choix est tout simplement impensable. De plus, beaucoup de communautés miskitus ne sont pas représentées, et les partisans des sandinistes en sont bien évidemment exclus. Par ailleurs, un grand nombre de participants sont des notables à la tête de réseaux d'influences militaires ou politiques. C'est bien évidemment le cas des comandantes. C'est aussi celui des " politiques ", qu'ils soient des laïcs, le plus souvent d'anciens comandantes, ou des religieux. Il faut enfin remarquer que si cette assemblée se termine par l'élection d'une nouvelle direction, cette désignation ne se fait pas à bulletin secret, mais au cours d'un vote public qui autorise des pressions sur les électeurs. Pourtant, quels que soient ces manquements à la démocratie, on n'en discerne pas moins différents bouleversements participant de mutations démocratiques. Les précédentes assemblées, comme celles réalisées lors de la fondation d'ACARIC ou d'ALPROMISU, étaient convoquées par un tout petit nombre de pasteurs, de prêtres ou de professeurs. Ceux-ci invitaient les participants à s'associer à une entreprise dont les objectifs étaient d'ores et déjà définis. Les assistants n'étaient invités qu'à ratifier des décisions préalablement prises, mais nullement à les discuter ou à les amender. Le fonctionnement de ces réunions fondatrices était calqué sur celui 
des commissions des paroisses de l'Eglise morave. A l'image du pasteur, les fondateurs invitent certes les participants à débattre et à discuter, mais ce dans un cadre au préalablement fixé, et où l'on discute des modalités de la mise en œuvre d'options décidées par un petit nombre. La chose n'avait guère été différente lors de la création de MISURASATA. L'assistance avait été soumise à la politique du fait accompli, tant pour ce qui était de la disparition d'ALPROMISU et de son remplacement par MISURASATA, que pour ce qui était de la désignation des dirigeants de cette nouvelle organisation.

Ici on procède à l'inverse. C'est un conclave de plusieurs dizaines de comandantes, de «politiques » et de représentants des réfugiés qui appellent à une assemblée plénière dont l'issue est dès le départ reconnue comme très incertaine. Plus de cinq cents participants y auront non seulement le droit de vote, mais fixeront aussi l'ordre du jour. On est ici fort loin du discours verticaliste qui caractérisa la fondation de MISURASATA. A Rus-Rus, les délégués accèdent et participent au détail des intrigues politiques. Les comandantes rapportent et exposent la situation politico-militaire des zones où ils combattent. Les représentants des réfugiés font de même. On assiste par ailleurs à de nombreux échanges de vue sur la possibilité ou non de continuer la guerre, sur le caractère aléatoire de l'aide nord-américaine, sur l'attitude du H.C.R., comme sur certaines offres de paix des sandinistes.

Les modalités de l'élection d'une direction politico-militaire sont là encore parfaitement nouvelles. Les dérives dictatoriales de Fagoth, comme celle de Rivera, sont non seulement dénoncées, mais plus fondamentalement la tenue même de cette assemblée constitue dès le départ une remise en cause de leurs pouvoirs respectifs. Une des premières décisions de l'assemblée est d'affirmer que ceux-ci «participent à la lutte comme (simples) membres mais plus comme dirigeants». Fait significatif, cette remise en question s'articule à la reconnaissance d'un vide du pouvoir et de la nécessité d'une désignation démocratique pour pallier une telle situation. On est loin d'une situation où une camarillafait un coup de force contre un groupe dirigeant et le remplace au pied levé. Deux commandants en chef sont contestés et déposés et l'on décide d'appeler des candidats à la direction politico-militaire à se faire connaître afin que les membres de l'assemblée soient à même de choisir entre plusieurs possibles équipes dirigeantes. C'est dire que pour la première fois dans l'histoire de la guérilla, on reconnaît que des divisions et des rivalités existent au sein des combattants. On admet que celles-ci peuvent s'exprimer publiquement et sont tranchées par l'addition de suffrages individuels. L'importance accordée au comptage des votes est de ce point de vue exemplaire. Les votants sont appelés un à un à exprimer leur préférence, tandis qu'une équipe de scrutateurs marque au tableau les choix exprimés. On est là aussi en rupture avec les idéaux unanimistes propres aux miskitus. Les miskitus professent en effet que les décisions légitimes sont celles prises en commun (asla bapanka) à partir d'une idée fédératrice (asla lukanka). L'idéal est un état d'harmonie entre les différentes parties composant la communauté (kupia kumi) ${ }^{10}$. Or, ici on procède à l'inverse. Comme dans les discussions au sein des assemblées villageoises ou des Eglises, les différences sont signifiées et les désaccords s'expriment. Mais, loin de chercher à ramener ces différends à un accord minima grâce à de multiples ajustements des propositions en présence, on choisit de matérialiser ces différences par une expression arithmétique. Et se fait jour l'idée neuve que l'addition mécanique des préférences exprimées individuellement permet d'adopter une option plutôt qu'une autre. Se fait aussi jour 
l'idée que la majorité gouverne pour un temps et qu'à l'issue de ce mandat une nouvelle assemblée sera à nouveau convoquée.

Il est une ultime manifestation du changement qu'il convient de souligner, la publicité des débats et leur enregistrement. En effet, différents journalistes de la presse hondurienne et internationale sont invités à assister aux débats. Parallèlement, certains guérilleros sont chargés de consigner les débats, les résultats des votes et les décisions prises par l'assemblée.

La généralisation des pratiques démocratiques

Les pratiques démocratiques instituées à Rus-Rus ne vont pas rester lettre morte mais s'imposer comme une nouvelle praxis. Ces nouvelles manières de faire et de penser se font ainsi sentir tant dans les relations avec les réfugiés, que dans la manière de conduire la guerre et de résoudre les affrontements au sein de la guérilla.

On a vu comment, dès avant l'assemblée de Rus-Rus, les réfugiés avaient été réorganisés par les agences du H.C.R. selon des patrons conformes aux canons de la démocratie représentative. On a décrit comment les réformateurs avaient accompagné ce mouvement en mettant en place un Comité central coiffant ces différentes juntes de gouvernement communautaire. La fondation de Kisan va coïncider avec une relance de ces initiatives. Les membres de la Commission politique et leurs assesseurs deviennent à la fois capables de proposer certaines actions de santé publique et d'éducation aux O.N.G. travaillant pour le H.C.R., comme de participer aux programmes agricoles lancés par ces dernières. Et l'on voit apparaître toute une stratégie incrémentaliste de la part des guérilleros à l'égard du H.C.R. Celui-ci n'est plus considéré a priori comme une agence favorable aux sandinistes, mais les membres de KISAN apprennent à tenir compte des règlements internationaux et à accepter que des positions de neutralité face au conflit qui les oppose aux sandinistes soient possibles. Ils découvrent aussi que les réfugiés miskitus et mayangnas puissent ne plus vouloir appuyer la guérilla comme ils l'avaient fait lors des années 1982-1983. Les guérilleros mettent ainsi fin au mode de conscription qu'ils avaient institué. Plus encore, ils vont s'avérer capables d'entendre les remontrances du H.C.R. au sujet des mayangnas. Considérés comme les membres d'une ethnie inférieure par la plupart des Miskitus, les Mayangnas ont été soumis au recrutement forcé, ce moins pour fournir des combattants que des porteurs aux guérilleros. Forts de l'appui d'un anthropologue allemand, un certain nombre d'entreeux dénoncent ces faits au H.C.R. et aux membres du Département d'État américain. Dès lors, s'ouvrira une véritable négociation entre les comandantes réformateurs, la Commission politique, le Conseil des anciens, le H.C.R. et les dirigeants mayangnas afin que de telles pratiques prennent fin.

Sitôt élue, la nouvelle direction politico-militaire doit faire face à un double problème. Démoralisés par la précarité de leur situation et la suspension de l'aide nordaméricaine, beaucoup de guérilleros désertent. Et, à bien des égards, les turbulences liées à l'expulsion de Fagoth ne font qu'accentuer le mouvement. Par ailleurs, sur le terrain à l'intérieur du Nicaragua, certains commandants sont devenus autant de roitelets incontrôlés qui multiplient les abus à l'encontre des populations civiles. Les réfugiés connaissent eux aussi une baisse de moral sans précédent, beaucoup en viennent à douter d'une possible victoire sur les sandinistes, ainsi que des possibilités du H.C.R. de les protéger. De telle manière que certains songent très sérieusement à demander un rapatriement. 
A la différence des méthodes qui avaient prévalu jusqu'alors, la direction politicomilitaire va choisir de faire front à ces difficultés par la négociation et la persuasion. Nul doute pourtant que certains abus de pouvoir persistent. Un commandant fidèle à Fagoth, Francisco Gonzalez, est ainsi emprisonné arbitrairement des semaines durant dans des conditions particulièrement abjectes ${ }^{11}$. Pourtant, premier signe du nouveau, il n'est pas exécuté. Et, devant les pressions du H.C.R., le nouvel État-major est obligé de le libérer et le laisse se réinstaller au milieu des réfugiés. Parallèlement, et ce là encore pour la première fois, les commandants vont laisser les guérilleros abandonner les bases et aller dans les camps de réfugiés. Leur raisonnement est double. Ils considèrent qu'ils ne sauraient légitimement maintenir durablement sous les armes des guérilleros qui manifesteraient la volonté d'abandonner la lutte. Ils affirment en même temps qu'une telle injustice est de plus contre-productive. De telles recrues sont le plus souvent particulièrement peu fiables militairement parlant. Enfin, quand de tels faits viennent à être connus, ils s'avèrent désastreux pour l'image de marque de la guérilla. Ainsi, des quelques 1300 guérilleros que compte KISAN dans ses bases honduriennes, près de 500 vont retourner à la vie civile et opter pour le statut de réfugié. Ceux qui décident de rester vont être réorganisés et motivés par de longues discussions avec les membres du nouvel Etat-major. Désormais, les guérilleros ne reçoivent plus seulement une instruction militaire mais aussi une formation politique. Et différents comandantes mettent l'accent sur la nécessité qui est la leur de respecter dorénavant les grandes lignes de la convention de Genève. Ils expliquent à leurs troupes comment c'est tout à la fois une obligation morale et un impératif stratégique face aux campagnes de presse des sandinistes et de leurs sympathisants.

Il est en même temps décidé de destituer certains comandantes, tel Pitufo,qui sont devenus autant de seigneurs de la guerre terrorisant les populations des zones où ils sont cantonnés. On envoie donc de nouveaux responsables acquis aux idéaux de la réforme les relever de leurs commandements. Une fois sur place, ceux-ci reprennent les troupes en main et chassent les plus corrompus. Ils ordonnent aussi la libération de prisonniers que certains traitaient de la façon la plus inhumaine, telle une infirmière et un médecin sandinistes. La première a été violée de façon répétée par la plupart des membres du groupe de Pitufo,tandis que le second a été littéralement traité comme un esclave. De même, est-il ordonné que certains jeunes recrutés de force dans les communautés soient rendus à la liberté. Il est aussi très nettement signifié que les jeunes filles venues souvent volontairement cuisiner et laver pour les guérilleros sont à tous moments libres de retourner dans leurs familles. Il est enfin précisé que personne n'est en droit d'abuser d'elles. Ce style nouveau va aussi faire sentir sa marque face aux offres de paix que font les sandinistes à certains comandantes, tel Panting puis Rubio dans la zone de Yulu. Ces propositions de cessez-le-feu sont à l'évidence très mal accueillies par le nouvel Etat-major. Les présents à l'assemblée de Rus-Rus viennent de décider de la poursuite de la guerre et les troupes ont été réorganisées à cet effet. Pourtant, quel que soit le sentiment de trahison que ressentent beaucoup des membres de KISAN face à la décision de Panting d'accepter les offres des sandinistes, on n'aura pas recours à l'assassinat comme autrefois pour faire rentrer dans le rang des opposants. Les nouveaux dirigeants vont bien évidemment multiplier les opérations militaires contre les sandinistes tout au long des négociations, mais ils ne vont $\mathrm{ni}$ attaquer le lieu des négociations, ni s'en prendre à leurs anciens compagnons d'armes. Et une fois la paix de Yulu conclue, ils la dénoncent certes comme un accord honteux, mais d'une certaine façon l'acceptent comme un élément de la nouvelle donne politico- 
militaire. C'est dire que l'on est loin de l'ambiance qui avait pu régner dans les années 1982-1983 où Fagoth n'hésitait pas à envoyer à la mort ses opposants, soit en montant des embuscades contre eux, soit en les envoyant combattre dans des zones où ils les savaient en situation d'infériorité notoire.

Ce choix de la délibération en commun, comme ce souci de la désignation démocratique des équipes dirigeantes vont faire sentir la force de leur emprise à l'occasion des crises dues aux malversations de certains membres des nouveaux organes de directions, comme aux accords de paix avec les sandinistes ou aux tentatives de retour en force des dirigeants évincés lors de l'assemblée de Rus-Rus. En effet, sitôt nommés, certains responsables détournent à leur profit les ressources fournies par les Etats-Unis, puis abandonnent leurs postes. En même temps, comme on l'a vu, certains comandantes ayant conclu des cessez-le-feu avec les sandinistes font sécession et créent leur propre mouvement, KISAN pro paz. Enfin, forts de leurs contacts avec les Nord-américains, d'autres secteurs de la Contra, Brooklyn Rivera et Steadman Fagoth multiplient les intrigues pour tenter de retrouver leur influence passée et parvenir à réoccuper les premières positions. Rentré en grâce auprès de l'armée hondurienne, ce dernier fait jeter en prison toute une partie de la direction de KISAN puis la fait expulser. C'est dire que quels qu'aient été les réformes et les changements promus en 1985, KISAN n'en passe pas moins par des difficultés considérables. Pourtant, jamais les solutions autoritaires de remises des anciens dirigeants ne parviennent à s'imposer. Ceux-ci, dans une dernière manœuvre pour retrouver leur influence, n'ont d'autre solution que de convoquer à une nouvelle assemblée.

Ainsi en juin 1986, au lendemain du vote par le congrès des États-Unis d'une aide de cent millions de dollars pour la Contra,Fagoth et Rivera obtiennent l'appui du Département d'État pour organiser une assemblée comme celle de Rus-Rus. Du 13 au 17 juin huit-cent quarante-six délégués, représentant cent six communautés, sont invités à débattre et à désigner de nouveaux organes dirigeants. Si les comandantes et les guérilleros sont présents, aucun d'entre-eux n'a le droit de voter. Au terme de trois jours de délibérations et de critiques des errements de Fagoth et de Rivera, les délégués des communautés décident néanmoins de leur redonner une chance. KISAN est dissout et remplacé par un nouveau mouvement YATAMA (Yapti Tasba Masraka nani Aslika/ Unidad de los pueblos indigenas de la madre patria). Le Conseil politique et l'Équipe politique sont remplacés par un Directoire, une Commission politique, une Commission des femmes et un Tribunal militaire (Fiscalía militar), où siègent trois personnes. Les trois membres qui siègent dans chacune de ces instances sont désignés à raison d'une personne par courant: les proches de Fagoth, ceux de Brooklyn, les comandantes réformateurs. Et quelques jours plus tard, les combattants seront réunis pour élire à leur tour les membres du nouvel Etat-major.

L'organisation de cette assemblée montre à l'évidence quelles sont les capacités de Fagoth et de Rivera à rebondir après avoir été destitués par les représentants des communautés et les guérilleros réformateurs. Il faut toutefois remarquer qu'il faudra toute la persuasion des représentants du Département d'Etat américain, et la promesse que cinq des cent millions d'aide à la Contravotés par le Congrès seront attribués aux guérilleros indiens, pour que les délégués acceptent la solution d'un triumvirat formé par Fagoth et Rivera et un représentant des réformateurs. Là encore, l'assemblée imposera pour une part ses vues puisqu'il n'était au départ question que de remettre en selle les dirigeants historiques et d'éliminer les réformateurs de tous les organes de 
direction. L'assemblée déclare aussi très solennellement qu'elle donne "une dernière chance à ces trois leaders, qu'ils doivent travailler unis et penser au peuple et non à leurs intérêts personnels ». Elle précise que « aucun d'entre eux ne peut assumer le commandement absolu de l'organisation, ce commandement est rotatif; ils n'ont pas davantage à assumer le commandement absolu des militaires parce que les combattants sont issus du peuple et (celuici) ne peut pas être utilisé comme troupe au service d'une seule personne $»^{12}$.

Par-delà cette mise en garde, l'un des nouveaux organes de pouvoir mis en place lors de cette assemblée, la Fiscalia militar, marque une préoccupation nouvelle pour les droits de l'homme. Une telle institution résulte à la fois des désirs des réformateurs, comme de certains membres du Département d'Etat, tous soucieux de poursuivre la lutte contre les crimes commis par certains comandantes et leurs fidèles. Il est d'ailleurs à noter que l'une des têtes de file des réformateurs, le comandante Mono, fait partie des dirigeants de cette institution. De même, procède-t-on à la mise en place d'une commission de femmes. Si l'on ne saurait s'illusionner sur le pouvoir d'influence de cette instance, cela n'en traduit pas moins un véritable bouleversement des mœurs au sein de la guérilla comme au sein des communautés miskitus. Enfin, il convient de souligner que les différents membres de l'Etat-major seront cette fois-ci élus par un conseil de comandantes. Et si Fagoth et Rivera ont certes le pouvoir de lancer des candidats, ils vont dans les faits souvent être obligés d'appuyer des candidats qui auront acquis une aura propre. Ainsi, le comandante Blas, élu à la tête de l'État-Major, le sera du fait de sa remarquable audace au combat et non parce que Fagoth l'a soutenu. De plus, après cette élection, certains des nouveaux responsables, par trop dépendants d'un des leaders de MISURASA et de MISURASATA, seront obligés de démissionner et seront remplacés par des réformateurs.

A retracer les différents moments de la mise en place de pratiques démocratiques au sein de la guérilla et de l'exil miskitu, on découvre combien cette invention fut non seulement paradoxale - elle surgit au beau milieu d'une guerre - mais combien elle résulta de la conjonction de différents projets. Elle ne fut en effet, ni l'œuvre d'un secteur miskitu porté par un même idéal, la réforme de la guérilla, ni une imposition par des agents extérieurs à la société miskitu. Cette entreprise se situe à la croisée de plusieurs expériences et de plusieurs projets, et résulte de leurs interactions.

Il est un premier moment de cette expérience qui renvoie aux nécessités fonctionnelles de la pratique de la guerre de guérilla. Celle-ci n'est possible qu'à condition de reconnaître une marge d'autonomie aux comandantes qui mènent leurs hommes au combat. Et une telle expérience vient mettre en doute la croyance en la toute puissance d'un dirigeant charismatique. Il en est un deuxième où l'on puise à la tradition chrétienne pour critiquer les abus de pouvoir des dirigeants de la guérilla. Si les sandinistes ont été comparés au Pharaon, Fagoth apparaît bientôt à certains comme un autre Pharaon.

Reprenant à leur compte la pratique des assemblées propres aux communautés miskitus, un certain nombre de combattants en changent l'assiette. S'ils renouent avec l'idée de la discussion en commun et de l'examen collectif, ils abandonnent celle de l'unité et admettent la division en groupes d'opinions antagonistes. Plus encore, ils reconnaissent que ces divisions sont momentanément tranchées par la pratique du vote majoritaire. Cette découverte va enfin être aiguillée par un certain nombre de critiques et de pressions externes. Celles d'observateurs de la guérilla, journalistes et anthropologues qui renforcent dans leurs convictions ceux pour qui le nom de 
" combattants de la liberté ${ }^{13}$ est un nom qui oblige à respecter certaines pratiques et à s'en interdire d'autres. La présence du H.C.R. sera, elle aussi, un atout considérable dans la lutte des réformateurs. Les membres de cette organisation internationale non seulement dénonceront certains abus de la guérilla, mais offriront une réelle protection à tous les membres de la guérilla persécutés par les puissants du moment.

\section{NOTES}

1. Cette étude constitue une suite à un premier travail sur la guérilla miskitu, "Comandantes, Etat-major et guérilleros : jeux de pouvoir à l'intérieur de la guérilla miskitu (Nicaragua 1981-1984) », Cahiers des Amériques latines, n³6, 2001/1. Comme dans l'étude précédente, je m'appuie d'une part sur des entretiens réalisés avec les guérilleros en 1984-1985, puis sur de nouveaux entretiens réalisés après leur retour à la vie civile, de 1997 à aujourd'hui, ainsi que sur différents documents d'archives de la guérilla qu'ils m'ont permis de consulter et de recopier. J'ai publié un de ces entretiens et un de ces documents dans la revue du Centre d'études mexicaines et centraméricaines (CEMCA), TRACE, $n^{\circ} 41$, juillet-aout 2002, Mexico, « Francisco Gonzalez-Yugo, un comandante runguero »; « Santiago Benjamin-Coyote, Reseña Histórica de la Lucha Indigena en Nicaragua' ».

2. . Je renvoie à deux travaux que j'ai consacrés à ces épisodes de l'histoire nicaraguayenne « Elections au Nicaragua : réaménagement du système des 'concurrents pour le pouvoir' » in Problèmes d'Amérique latine, $\mathrm{n}^{\circ} 2$, Juillet-Septembre, 1991, pp. .21-40 ; et « Réflexions sur l'action armée et la constitution d'acteurs politicomilitaires : contre et recontras nicaraguayens 1982-1993 » in Cultures \& Conflits, ${ }^{\circ} 12$, Hiver 1993-1994.

3. . Je résume ici deux études antérieures que j'ai consacrées à ce sujet, « Moskitia nicaraguayenne 1979-1999 : changements sociaux et égalisation des conditions » in Joel Delhom et Alain Musset, Nicaragua dans l'œil du cyclone, IHEAL-IHNCA_UBS, paris 2000 et « Cambios culturales y sociopoliticos en las comunidades mayangnas y miskitus del Rio Bocay y del Alto Coco (1979-2000) » in Journal de la Société des Américanistes, tome 87, 2001, pp. 376-391. On trouvera par ailleurs un très remarquable tableau de la société miskitu jusque dans les années 1970 dans les travaux de Mary Helms W., Asang : adaptaciones al contacto cultural en una sociedad misquito, (trad. de l'anglais, 1971), Instituto Indigenista Interamericano, Mexico, 1976 ; Middle America : A Cultural History of Heartland and Frontier, Englewood Cliffs, Prentice Hall 1975 ; « The Cultural Ecology of a Cultural Tribe ", Ethnology, vol. VIII, n 1969.

4. . J'emprunte cette expression à Michel de Certeau. Cf. La prise de parole. Pour une nouvelle culture, Paris, Desclée de Brower, 1968.

5. . Cf. l'article cité en note 1.

6. . On trouvera dans l'article de Ramon Vera H., «Acercar el mar a los peces » (in Poder y Cultura de la Violencia, sous la coordination de Susana B.C. Devalle, Colegio de Mexico, Mexico 2001), un excellent témoignage sur l'état d'esprit qui pouvait prévaloir chez les membres des ONG travaillant dans les camps du HCR au Honduras. 
7. . Courant 1982 certains comandantes se sont rebellés contre Fagoth et se sont ralliés au commandement de Brooklyn Rivera qui a monté au Costa Rica un Etat-Major concurrent de celui de Fagoth.

8. . Jusqu'à 700 selon certaines estimations de différents participants.

9. . Le Directorio político comprend cinq membres, le Consejo de Ancianos huit, le Consejo político quatre, l'Equipo político neuf et l'Etat-major sept.

10. . Je reprends ici un passage d'une étude antérieure, « Moskitia nicaraguayenne 1979-1999 changements sociaux et égalisation des conditions », op.cit.

11. . Cf. Gilles Bataillon, «Francisco Gonzalez-Yugo, un comandante runguero », op. cit. 12. . « ninguno de ellos tiene mando absoluto sobre la organizacion pero que podrian tomar mandos rotativos; que no tienen (mando absoluto) sobre los militares porque los combatientes son del pueblo y (este) no puede ser usado como tropa de una persona ». Cf. Gilles Bataillon, « Santiago Benjamin-Coyote, 'Resena Historica de la Lucha Indigena en Nicaragua' », op. cit.

13. . Le terme est de Ronald Reagan et sera rapidement repris par les contras.

\section{RÉSUMÉS}

Depuis 1989, date de la fin de la guerre civile contras/sandinistes et de la victoire de l'opposition sur le Front sandiniste de libération nationale (FSLN), les Miskitus (indiens du Nicaragua), font l'expérience à tous les niveaux de la vie sociale de formes de pouvoir démocratiques. La découverte et l'apprentissage de pratiques et de mœurs démocratiques par les Miskitus tiennent sans nul doute à " l'esprit du temps ». Pourtant, cette découverte et cet apprentissage ont aussi été des expériences propres aux Miskitus qu'il convient de retracer d'autant qu'ils prennent place dans un contexte qui leur était a priori peu favorable, celui de l'action armée et de la guerre civile. C'est ce que cet article entreprend de faire en prenant appui d'une part sur des entretiens réalisés avec les guérilleros miskitu en 1984-1985, puis sur de nouveaux entretiens réalisés après leur retour à la vie civile, de 1997 à aujourd'hui, ainsi que sur différents documents d'archives de la guérilla.

The Miskitus Indians from Nicaragua are since the 1989 end of the contras-sandinists civil war and the victory of the opposition on the sandinist national liberation front (SNLF) experiencing democratic forms of power at every stage of social life. The discovery and the apprenticeship of democratic practices and manners surely come from the « spirit of time ». But this discovery and apprenticeship have also been the Miskitus' sole experience, which one ought to retrace in so much as it occurred in the a priori unfavourable context of armed battles and civil war. This article intends to do so, supported on the one hand of interviews made with miskitu guerrilleros in 1984-1985 and others from 1997 to today, after their return to civil life, and on the other hand on different guerrilla archive documents. 
INDEX

Index géographique : Amérique centrale, Nicaragua

Mots-clés : anthropologie, démocratie, guerre

Index chronologique : 1980 - 1990 